\title{
Estudo psicométrico preliminar do Inventário Dimensional dos Transtornos da Personalidade (IDTP)
}

\author{
Lucas de Francisco Carvalho \\ Universidade São Francisco
}

Ricardo Primi ${ }^{1}$

Universidade São Francisco

\begin{abstract}
RESUMO
O presente estudo teve como objetivo desenvolver um instrumento para avaliação dos transtornos da personalidade e buscar por evidências de validade baseadas na estrutura interna desse instrumento. $\mathrm{O}$ instrumento desenvolvido, o Inventário Dimensional de Transtornos da Personalidade (IDTP), foi aplicado em 350 participantes, entre 18 e 67 anos, de ambos os sexos. Foram realizadas análises exploratórias por componentes principais de primeira e de segunda ordem, correlações entre as escalas do instrumento, e calculados os coeficientes alfa de Cronbach. De acordo com a literatura utilizada e especificamente as categorias diagnósticas para transtornos da personalidade, a maior parte dos fatores hipotetizados para o teste foi encontrada, e os coeficientes alfa variaram entre 0,65 e 0,89 . Além disso, a estrutura fatorial de segunda ordem, composta por três fatores, foi coerente de acordo com a literatura prévia.

Palavras-chave: Transtornos psiquiátricos; escala de autorrelato; traços de personalidade; DSM.
\end{abstract}

\begin{abstract}
Preliminary psychometric study of the Personality Disorders Dimensional Inventory (IDTP)

This study aimed to develop an instrument for personality disorders assessment and seek for validity evidence based on the internal structure of this instrument. The developed instrument, the Dimensional Inventory of Personality Disorders (IDTP), was applied to 350 participants, from 18 to 67 years, of both sexes. First and second order exploratory analyzes by principal components were performed, correlations between the scales of the instrument, and calculated the alpha's Cronbach coefficients. According to the literature and specifically the diagnostic categories for personality disorders, most hypothesized factors were found for the test and the alpha coefficient ranged between .65 and .89 . Furthermore, the secondorder factor structure, consisting of three factors, was consistently according to prior literature.
\end{abstract}

Keywords: Psychiatric disorders; Self-report scale; personality traits; DSM.

O estudo da personalidade, e mais especificamente da avaliação desse construto configura-se atualmente como um dos mais amplos campos de estudo da psicologia (Carvalho \& Ambiel, 2010; Groth-Marnat, 2003; John, Robins \& Pervin, 2008). A teoria dos estilos da personalidade de Theodore Millon é um exemplo a ser observado, cuja avaliação se encontra indicada aos aspectos saudáveis e patológicos da personalidade (Alchieri, 2004; Millon \& Davis, 1996), e que apresenta robustez teórica (Millon, 2011).

Millon utiliza-se de três esferas que são baseadas na psicologia evolutiva, nomeadas de fases evolutivas: Orientações para Existência, Modos de Adaptação, e Estratégias para Replicação (Millon \& Davis, 1996; Davis, 1999; Millon, Millon, Meagher, Grossman, Ramanath, 2004; Strack \& Millon, 2007). A primeira fase,
Orientações para Existência, está relacionada à tendência da pessoa a expressar mecanismos que favoreçam a busca pelo prazer (foco na busca por experiências prazerosas e ganhos) e a evitação da dor (foco na evitação de ações ou situações que sejam perigosas e tragam danos). Uma vez que o indivíduo esteja orientado, ele precisa manter sua existência por meio de uma complexa relação com o ambiente (Modos de Adaptação).

A segunda fase evolutiva está relacionada aos modos de se adaptar que tornam as trocas entre indivíduo e ambiente possíveis. Algumas pessoas tendem a modificar o ambiente ao redor (tendência ativa) e outros são mais propensos a acomodar-se ao ambiente em que vivem (tendência passiva). Após a adaptação ao ambiente em que vive, a fase evolutiva Estratégias de Replicação trata da continuidade do indivíduo, que é limitada pelo 
tempo. Esta fase evolutiva diz respeito às estratégias desenvolvidas pelas pessoas para ultrapassar a limitação da própria existência, que podem ser de autopropagação (indivíduos mais voltados para o eu) ou estratégias voltadas para o cuidado da prole (indivíduos mais voltados aos outros). Foge do escopo do presente estudo apresentar minuciosamente a teoria de Millon, para aprofundamento, sugere-se recorrer à obra do autor (Millon \& Davis, 1996; Millon et al., 2004; Millon, 2011).

Em decorrência das três fases evolutivas, Millon derivou 15 estilos patológicos da personalidade, sendo eles, esquizoide, esquizotípico, paranoide, borderline, antissocial, narcisista, histriônico, evitativo, dependente, compulsivo, negativista, sádico, masoquista, hipomaníaco e depressivo (Davis, 1999; Millon \& Davis, 1996; Millon et al., 2004). Um estilo patológico da personalidade pode-se configurar como um transtorno da personalidade, quando estratégias alternativas empregadas para atingir objetivos, se relacionar com os outros, ou lidar com o stress são poucas e rígidas (inflexibilidade adaptativa); quando percepções habituais, necessidades, e comportamentos perpetuam e intensificam dificuldades pré-existentes (círculos viciosos); e, quando a pessoa tende a uma baixa resiliência frente a condições psicostressoras (estabilidade tênue).

A relevância da avaliação dos transtornos da personalidade na clínica é considerada por diversos autores (Carvalho \& Primi, 2013; Handler \& Meyer, 1997; John, Robins \& Pervin, 2008; Strack \& Millon, 2007; Widiger \& Trull, 2007). A despeito dessa importância, não há consenso de como esses transtornos podem ser melhor diagnosticados. Baseado em sua teoria, Millon e colegas desenvolveram um instrumento de autorrelato para avaliação dos transtornos da personalidade, o Millon Clinical Multiaxial Inventory (MCMI; Millon, Millon \& Davis, 1994; Millon et al., 2004). A versão mais atual do MCMI, o MCMI-III, é composta por 175 itens, distribuídos em 28 escalas distintas, sendo 14 dessas escalas para avaliação dos transtornos da personalidade. No que diz respeito às propriedades psicométricas do MCMI-III, uma série de estudos são encontrados na literatura internacional (Craig \& Bivens, 1998; Dyce, O'Connor, Parkins \& Janzen, 1997; Millon et al., 1994; Millon \& Davis, 1996; Rossi, Ark \& Sloore, 2007; Rossi, Brande, Tobac, Sloore \& Hauben, 2003; Strack \& Millon, 2007).

O manual do MCMI-III (Millon et al., 1994) apresenta os índices de fidedignidade encontrados, por meio de uma aplicação em 358 pacientes, para as escalas do instrumento. Os índices de consistência interna (coeficiente alfa) excederam 0,80 em pelo menos 20 escalas do teste. Contudo, especificamente no que concerne às escalas de avaliação dos transtornos da personalidade, observou-se que as escalas Narcisista e Compulsivo apresentaram coeficientes inferiores a 0,70 . São encontrados também estudos que apontam para soluções fatoriais das escalas de transtornos da personalidade do MCMI-III, cujos principais resultados estão disponibilizados na Tabela 1. Vale ressaltar que para os três estudos apresentados na tabela, os dados encontrados foram procedidos com base na análise por componentes principais, utilizando como critério para retenção dos fatores a análise paralela (Rossi, Ark \& Sloore, 2007), o scree plot, eigenvalue superior a 1 e análise paralela (Dyce et al., 1997) e eigenvalue superior a 1 (Craig \& Bivens, 1998).

Como pode ser verificado, diferentes soluções fatoriais foram encontradas nos três estudos apresentados. No geral, alguns padrões são recorrentes ao longo dos dados encontrados: um fator abarcando as escalas Depressivo, Dependente, Negativista, Masoquista, Esquizotípico, e Borderline; um fator que, entre outras, é composto pelas escalas Antissocial e Sádico; e, por fim, cargas negativas para a escala Compulsivo. Por um lado, os agrupamentos das escalas foram coerentes de acordo com o funcionamento da personalidade que cada uma avalia. Por outro, é importante ressaltar que a teoria de Millon não foi concebida com base no agrupamento fatorial e, por isso, não existem expectativas $a$ priori determinadas em relação ao que deve ser encontrado a partir da realização de análises fatoriais (Strack \& Millon, 2007). Assim, apesar dos padrões evidenciados nos estudos, não há consenso entre os autores de qual seria a solução fatorial mais adequada. Os dados apresentados na literatura podem ser considerados como adequados em relação às propriedades psicométricas do MCMI-III, apesar de algumas inconsistências, sobretudo acerca do número de dimensões propostas pela teoria de Millon e o número de fatores encontrados em análises fatoriais (Craig \& Olson, 2001; Haddy, Strack \& Choca, 2005; Millon et al., 1994; MullinsSweatt \& Widiger, 2007).

Em uma revisão da literatura nacional, foi encontrado apenas um instrumento aprovado pelo Conselho $\mathrm{Fe}$ deral de Psicologia ([CFP]; vide satepsi.cfp.org.br/), o Psychopathy Checklist Revised (PCL-R; Morana, 2003), validado para uma amostra brasileira, que tem como objetivo avaliar o funcionamento psicopata, alta- 
Tabela 1

Resultados das Análises Fatoriais de Segunda Ordem com o MCMI-III

\begin{tabular}{lll}
\hline Estudos & Número de Fatores & \multicolumn{1}{c}{ Distribuição } \\
\hline $\begin{array}{l}\text { Rossi, Ark e Sloore } \\
\text { (2007) }\end{array}$ & Três fatores & $\begin{array}{l}\text { 1- Depressivo, Dependente, Negativista, Masoquista, Esquizotípico e Borderline } \\
\text { 2- Antissocial, Sádico e Compulsivo (carga negativa) } \\
\text { 3- Histriônico, Narcisista, Esquizoide (carga negativa) e Evitativo (carga negativa) }\end{array}$ \\
& & 1- Evitativo, Depressivo, Dependente, Negativista, Masoquista, Esquizotípico e Borderline \\
Dyce e cols. (1997) & Quatro Fatores & $\begin{array}{l}\text { 2- Narcisista, Antissocial, Sádico e Paranoide } \\
\text { 3- Esquizoide e Histriônico (carga negativa) }\end{array}$ \\
& & 4- Compulsivo \\
\hline & 1- Esquizoide, Evitativo, Depressivo, Dependente, Histriônico (carga negativa), Narcisista \\
(carga negativa), Compulsivo (carga negativa), Masoquista e Borderline \\
(1998)
\end{tabular}

mente relacionado com um dos transtornos da personalidade descritos no DSM-IV-TR (APA, 2003) e DSM-5 (APA, 2013), o transtorno antissocial da personalidade. Esse dado sugere que, apesar da importância da avaliação e diagnóstico dos transtornos da personalidade, são escassos instrumentos validados nacionalmente para avaliação desses transtornos. Assim, considerando também a robustez da teoria de Millon (Craig, 1999; Davis, 1999; Strack \& Millon, 2007), o presente estudo teve como objetivo desenvolver um instrumento para avaliação dos transtornos da personalidade, bem como buscar por evidências de validade baseadas na estrutura interna desse instrumento.

\section{MÉTODO}

Este tópico será subdividido em duas etapas distintas, são elas: Etapa I, construção do instrumento; e, Etapa II, busca por evidências de validade baseadas na estrutura interna, e investigação da fidedignidade das escalas do instrumento desenvolvido na Etapa I.

\section{Etapa I - Construção do Instrumento}

\section{Procedimentos}

$\mathrm{Na}$ etapa inicial, o objetivo foi desenvolver um instrumento capaz de acessar os quatorze protótipos de transtornos da personalidade propostos por Millon (Millon et al., 2004). Por isso, primeiramente, definiuse claramente os construtos a serem mensurados (Gunther, 1999), sejam eles, os transtornos da personalidade esquizoide, evitativo, depressivo, dependente, histriônico, narcisista, antissocial, sádico, compulsivo, negativista, masoquista, paranoide, esquizotípico e borderline.
Para elaboração dos itens recorreu-se basicamente aos conceitos dos transtornos da personalidade descritos no DSM-IV-TR, à literatura específica da proposta teórica de Millon, e a outras referências que contribuíram para o entendimento dos transtornos da personalidade (APA, 2003; Beck, Freeman \& Davis, 2005; Caballo, 2007; Carlat, 2006; Millon, 1986; Millon et al. 1994; Millon \& Davis, 1996; Millon et al., 2004).

Em um primeiro momento, foram desenvolvidos 196 itens para avaliar os quatorze transtornos da personalidade de acordo com a teoria de Millon e os conceitos operacionalizados. Foram selecionados 42 itens (3 itens para avaliação de cada transtorno) entendidos como aqueles mais representativos do funcionamentochave, de acordo com a teoria, para cada transtorno da personalidade. Na sequência, foram selecionados mais 2 itens para cada uma das 14 escalas, que deveriam acessar características menos centrais dos transtornos, portanto mais periféricas, possivelmente mais passíveis de overlapping entre as escalas do instrumento, resultando em um total de 70 itens.

Os pesquisadores realizaram alguns refinamentos e revisões dos itens do instrumento, e sua versão final ficou composta por 92 itens para avaliação dos transtornos da personalidade. Os itens foram distribuídos em 14 escalas da seguinte maneira: Escala Esquizoide (7 itens), Escala Evitativo (7 itens), Escala Depressivo (7 itens), Escala Dependente (6 itens), Escala Histriônico (6 itens), Escala Narcisista (6 itens), Escala Antissocial (6 itens), Escala Sádico (6 itens), Escala Compulsivo (6 itens), Escala Negativista (7 itens), Escala Masoquista (7 itens), Escala Paranoide (7 itens), Escala Esquizotípico ( 7 itens), e Escala Borderline ( 7 itens). 
O conjunto de itens foi ordenado de forma randômica para a versão final do instrumento, contudo, tomouse o cuidado para que os primeiros itens a serem respondidos não fossem aqueles itens cuja avaliação dos respondentes poderia ser comprometida de alguma maneira (como "Já pensei em me suicidar." ou "Em situações ruins eu já me feri propositalmente.”). Assim, para os itens iniciais, foram selecionados aqueles avaliados pelos pesquisadores como "neutros" ou "positivos", como "Gosto de usar roupas que chamam atenção." e "Eu me considero uma pessoa muito especial.".

As respostas aos itens do instrumento, nomeado de Inventário Dimensional de Transtornos da Personalidade (IDTP), foram disponibilizadas em uma escala tipo Likert de 4 pontos, variando entre "Não me descreve" (1) e "Me descreve extremamente ou totalmente" (4). No cabeçalho do instrumento são requeridas as seguintes informações do respondente: inicias do nome, data de nascimento, e-mail, sexo, a realização de tratamentos psiquiátrico e psicológico, bem como o tempo de realização, e uso e tempo de medicação psiquiátrica. Entre o cabeçalho e os itens do IDTP, foram redigidas as instruções para responder o instrumento, juntamente com um exemplo de como o respondente deve proceder ao responder os itens do instrumento. Exemplos de itens do IDTP, são, "Evito participar de atividades em grupo, porque posso ser criticado.", "Fico irritado com as pessoas que acham que podem fazer as coisas melhor do que eu." e "Normalmente as pessoas não são confiáveis.".

Por último, para investigação de possíveis erros na digitação ou gramaticais e sintáticas dos itens, cabeçalho e instruções do IDTP, o instrumento foi aplicado em 30 participantes, todos com idade superior a 18 anos e graduandos do curso de Psicologia de uma universidade da cidade de São Paulo. Os participantes foram instruídos para que, além de responderem o instrumento, buscassem e apontassem possíveis dificuldades no processo de resposta e entendimento acerca do IDTP. Nenhum dos participantes relatou dificuldades para responder o instrumento. Por isso, finalizada esta etapa, deu-se início para a Etapa II da pesquisa.

Etapa II - Evidências de Validade Baseadas na Estrutura Interna, e investigação da fidedignidade das escalas do instrumento.

\section{Participantes}

Para esta etapa do estudo utilizou-se uma amostra de conveniência, de modo que foram recrutados 350 parti- cipantes, cujas idades variaram entre 18 e 67 anos $(\mathrm{M}=27,02 ; \mathrm{DP}=10,13)$, sendo $71,7 \%(\mathrm{~N}=251)$ do sexo feminino. Dos 350 participantes, 290 eram universitários dos cursos de Psicologia $(\mathrm{N}=158)$, Arquitetura $(\mathrm{N}=68)$, e Administração $(\mathrm{N}=64)$ de uma cidade do interior de São Paulo. Os outros 60 participantes eram pacientes psiquiátricos de uma clínica de uma cidade do interior de São Paulo. Entre os 290 universitários, 14 foram caracterizados como psiquiátricos por terem respondido afirmativamente às variáveis "fazer tratamento psiquiátrico" e "tomar remédio psiquiátrico", isto é, responderam estar em atendimento psiquiátrico atualmente e usar algum tipo de medicação psiquiátrica.

\section{Instrumentos}

Foi aplicado o Inventário Dimensional de Transtornos da Personalidade (IDTP) para avaliação das características típicas dos transtornos da personalidade. Trata-se de um teste de autorrelato composto por 92 itens para avaliação dos funcionamentos esquizoide, evitativo, depressivo, dependente, histriônico, narcisista, antissocial, sádico, compulsivo, negativista, masoquista, paranoide, esquizotípico e borderline. Os itens foram respondidos em uma escala tipo Likert de 4 pontos, variando entre "Não me descreve" (1) e "Me descreve extremamente ou totalmente" (4). O tempo médio para responder o IDTP foi de 15 minutos.

\section{Procedimentos}

Este estudo foi submetido e aprovado por um Comitê de Ética em Pesquisa (CAAE: 0144.0.142.00007). Para todos os participantes da pesquisa foi entregue o Termo de Consentimento Livre e Esclarecido (TCLE), que apresentava o objetivo principal deste estudo, bem como informava os participantes acerca da divulgação dos resultados, de acordo com as normas éticas. Assim, somente após concordar e assinar o TCLE, os participantes foram habilitados a participar deste estudo.

Foi aplicado um instrumento, o IDTP, desenvolvido na etapa I deste estudo. O IDTP é um instrumento para avaliação dos transtornos da personalidade de acordo com a teoria de Millon. Como já descrito anteriormente, trata-se de um inventário composto por 100 itens, distribuídos em 14 escalas distintas, representando os transtornos da personalidade. O tempo aproximado da aplicação foi de 20 minutos.

Foram realizadas análises exploratórias por componentes principais de primeira e de segunda ordem para os fatores primários encontrados, e foram investigadas 
as correlações entre as escalas do instrumento. No caso da análise de primeira ordem, foi utilizada uma rotação ortogonal, varimax, já que as correlações entre os fatores não justificou proceder a uma rotação oblíqua; diferente, para a análise de segunda ordem, a correlação entre os fatores encontrados justificou o uso da rotação oblíqua, e a mesma foi procedida (oblimin). Vale ressaltar que, como apontam Tabachnick e Fidell (1996), é possível a realização dessa análise a partir de 300 casos. $\mathrm{Na}$ sequência, foram calculados os coeficientes alfa de Cronbach, visando verificar a fidedignidade das escalas do instrumento. Para todas as análises realizadas foi utilizado o software estatístico SPSS versão 13.

\section{RESULTADOS}

Com o objetivo de explorar a estrutura dimensional do IDTP, as pontuações dos participantes foram submetidas a uma análise por componentes principais. Para verificação da adequação da amostra à análise, foi empregada a medida de adequação da amostra de KaiserMeyer-Olkin (KMO) e o teste de esfericidade de Bartllet. O KMO foi de 0,89 , indicando uma boa adequação dos dados à análise por componentes principais, e o teste de esfericidade de Bartlett foi significativo ao nível de 0,001 ( $\left.\chi^{2}=15500,986 ; \mathrm{gl}=4095\right)$, mostrando que houve correlações suficientes entre as variáveis para o emprego da análise.

Foram verificadas se as correlações entre os fatores justificavam o uso de uma rotação oblíqua. A maior parte das magnitudes das correlações foram inferiores a 0,20 , por isso, procedeu-se a uma rotação ortogonal. A extração dos fatores foi realizada por meio da análise dos componentes principais e rotação varimax. Foram incluídos apenas itens que apresentaram carga fatorial igual ou superior a 0,3 (Tabachnick \& Fidell, 1996).

Na sequência, verificou-se o scree plot para determinação da solução fatorial mais adequada, que apontou para uma composição de 4 fatores. Entretanto, buscouse recuperar os estilos originalmente propostos para o instrumento e, por isso, adotou-se um critério mais generoso quanto ao número de fatores. Originalmente, foram obtidos 23 fatores com eigenvalues acima de 1 , capazes de explicar $66,1 \%$ da variância total, sendo que o primeiro fator explicou $22,6 \%$ da variância total. Todos os fatores encontrados foram submetidos a uma análise de conteúdo dos itens de cada um dos 23 fatores, a fim de investigar se os fatores eram interpretáveis, isto é, se recuperavam as escalas teoricamente esperadas. Dos 23 fatores, os 15 primeiros fatores fo- ram interpretáveis de acordo com a teoria de Millon. Esses fatores se referiam aos seguintes estilos da personalidade: depressivo (fator 1); esquizoide (fator 2); borderline (fator 3); paranoide (fator 4); sádico (fator 5); compulsivo (fator 6); masoquista (fator 7); antissocial (fator 8); dependente (fator 9); histriônico (fator 10); negativista (fator 11); esquizotípico (fator 12); esquizotípico (fator 13); histrionnico (fator 14); e, masoquista (fator 15). $\mathrm{O}$ número de itens, as cargas fatoriais mínimas e máximas, os eigenvalues e o coeficiente alfa dos 15 fatores podem ser visualizados na Tabela 2 .

Pode-se observar que a carga fatorial dos itens variou entre 0,802 e 0,311 , o eigenvalue entre 20,63 (primeiro fator) e 1,27 (décimo quinto fator), e os coeficientes alfa entre 0,89 (primeiro fator) e 0,54 (décimo quinto fator). Na sequência, para a versão final do instrumento, tentando sempre manter a estrutura dimensional encontrada empiricamente, foram realizados ajustes de acordo com quatro critérios. São eles: correlações entre os 15 primeiros fatores encontrados na análise por componentes principais com os 14 fatores esperados teoricamente (para tanto, foram calculados tanto os fatores encontrados empiricamente quanto os teóricos); correlações entre os 15 fatores encontrados empiricamente; alterações nos índices de fidedignidade dos fatores por meio da exclusão de itens; alterações nos índices de fidedignidade de fatores com pouca representabilidade (baixo número de itens) com a inserção de itens teoricamente coerentes no fator. A Tabela 3 apresenta as correlações entre os 15 primeiros fatores empíricos encontrados com os 14 fatores teóricos esperados.

Pode-se observar que grande parte das correlações encontradas foram significativas ao nível de 0,01 . Exceção a isso foram as correlações dos fatores $1,2,7,11$ e 15 com o fator histriônico (significativa ao nível de $0,05)$, dos fatores 6 e 14 com o fator dependente, do fator 6 com o fator antissocial, dos fatores 5, 9, 12 e 15 com o fator compulsivo, do fator 14 com o fator negativista, dos fatores 6 e 14 com o fator masoquista, do fator 14 com os fatores paranoide, esquizotípico e borderline.

Verificou-se, ainda, que diferentes fatores empíricos (mensurando um mesmo construto) apresentaram magnitudes altas de correlação com os fatores teóricos esperados: fatores 7 e $15 \mathrm{com}$ masoquista; fatores 10 e 14 com histriônico; e, fatores 12 e 13 com esquizotípico. Por isso, as correlações entre esses fatores foram calculadas: $7 \times 15, \mathrm{r}=0,53, \mathrm{p}<0,001 ; 10 \times 14, \mathrm{r}=0,28$, $\mathrm{p}<0,001 ; 12 \times 13, \mathrm{r}=0,68, \mathrm{p}<0,001$. Os fatores 7 e 15 e 
Tabela 2

Fatores obtidos na Análise por Componentes Principais, eigenvalues e coeficientes alfa

\begin{tabular}{ccccc}
\hline Fator & No Itens & Cargas & Eigenvalue & $\begin{array}{c}\text { Coeficiente } \\
\boldsymbol{\alpha}\end{array}$ \\
\hline 1 & 17 & $0,324-0,725$ & 20,63 & 0,89 \\
2 & 16 & $0,312-0,704$ & 5,68 & 0,86 \\
3 & 10 & $0,311-0,654$ & 3,74 & 0,79 \\
4 & 9 & $0,333-0,673$ & 3,05 & 0,77 \\
5 & 7 & $0,383-0,755$ & 2,17 & 0,76 \\
6 & 6 & $0,432-0,792$ & 2,05 & 0,77 \\
7 & 7 & $0,354-0,675$ & 1,96 & 0,80 \\
8 & 8 & $0,323-0,802$ & 1,69 & 0,73 \\
9 & 7 & $0,323-0,802$ & 1,67 & 0,68 \\
10 & 4 & $0,324-0,728$ & 1,62 & 0,69 \\
11 & 7 & $0,304-0,652$ & 1,58 & 0,73 \\
12 & 5 & $0,321-0,715$ & 1,43 & 0,71 \\
13 & 4 & $0,395-0,662$ & 1,33 & 0,69 \\
14 & 5 & $0,328-0,781$ & 1,30 & 0,60 \\
15 & 4 & $0,329-0,362$ & 1,27 & 0,54 \\
\hline
\end{tabular}

os fatores 12 e 13 apresentaram correlações com magnitudes moderadas e significativas entre si, bem como altos índices de fidedignidade (conjunto de itens dos fatores 7 e 15 , alfa $=0,81$; conjunto de itens dos fatores
12 e 13 , alfa $=0,76)$ e maior representabilidade do construto com a união dos itens desses fatores. Por isso, com base na coerência teórica e relações estatísticas apresentadas, os itens dos fatores 7 e 15 (fator masoquista) foram unidos, assim como dos fatores 12 e 13 (fator esquizotípico). Na continuidade, na Tabela 4 são apresentadas as correlações entre os primeiros 15 fatores empíricos encontrados, que foram interpretáveis, com os oito fatores empíricos não interpretáveis (16 ao 23).

Como pode ser observado, a maior parte das correlações foram significativas ao nível de 0,01 . Assim, optou-se por destacar as correlações com magnitude igual ou superior a 0,50 (em negrito na Tabela 4). Partindo dessas correlações alguns fatores foram unidos e o coeficiente de fidedignidade dos novos fatores foi calculado. Como resultado, verificou-se que apenas a correlação entre o fator 3 e fator 19 aumentou o coeficiente de fidedignidade do conjunto de itens (de 0,79 para 0,82 ), potencializando também a representabilidade do construto. Uma vez que houve coerência teórica na união desses fatores, os itens "i100" e "i30" (fator 19) foram incluídos no fator 3.

Na continuidade, foi verificada se a exclusão ou inserção de itens em determinados fatores aumentariam o coeficiente de fidedignidade, a potencial capacidade de

Tabela 3

Coeficientes de Correlação de Pearson entre os fatores empíricos e os teóricos do IDTP

\begin{tabular}{|c|c|c|c|c|c|c|c|c|c|c|c|c|c|c|c|}
\hline Fatores & F1 & F2 & F3 & F4 & F5 & F6 & F7 & F8 & F9 & F10 & F11 & F12 & F13 & F14 & F15 \\
\hline Esquizoide & $0,67\left(^{* *}\right)$ & $0,92\left(^{* *}\right)$ & $0,35\left(^{* *}\right)$ & $0,52(* *)$ & $0,33\left({ }^{* *}\right)$ & $0,22\left(^{* *}\right)$ & $0,59\left(^{* *}\right)$ & $0,23\left({ }^{* *}\right)$ & $0,39\left(^{* *}\right)$ & $0,29\left(^{(*}\right)$ & $0,58\left(^{* *}\right)$ & $\left.0,411^{* *}\right)$ & $0,45\left(^{* *}\right)$ & $-0,14\left({ }^{* *}\right)$ & $0,48\left(^{* *}\right)$ \\
\hline Evitativo & $\left.0,70{ }^{* *}\right)$ & $\left.0,811^{* *}\right)$ & $0,69\left(^{* *}\right)$ & $0,52\left(^{* *}\right)$ & $\left.0,30{ }^{* *}\right)$ & $0,23\left({ }^{* *}\right)$ & $0,75\left(^{* \star}\right)$ & $\left.0,211^{* *}\right)$ & $0,58\left(^{* *}\right)$ & $0,33\left(^{* *}\right)$ & $0,68\left(^{* \star}\right)$ & $\left.0,50{ }^{* *}\right)$ & $0,48\left(^{* *}\right)$ & $-0,18\left({ }^{* *}\right)$ & $0,58\left(^{* *}\right)$ \\
\hline Depressivo & $0,93\left(^{* *}\right)$ & $0,76\left(^{* *}\right)$ & $0,450\left(^{* \star}\right)$ & $0,55\left(^{* *}\right)$ & $0,33\left(^{* *}\right)$ & $0,18\left(^{* *}\right)$ & $0,65\left(^{* *}\right)$ & $\left.0,211^{* *}\right)$ & $0,47\left(^{\star *}\right)$ & $0,29\left(^{* *}\right)$ & $\left.0,73^{(* *}\right)$ & $0,42\left(^{* *}\right)$ & $0,47\left(^{* \star}\right)$ & $-0,17\left({ }^{* *}\right)$ & $0,54\left({ }^{* *}\right)$ \\
\hline Dependente & $\left.0,58^{(* *}\right)$ & $0,52\left(^{* *}\right)$ & $\left.0,511^{* *}\right)$ & $0,37\left(^{* *}\right)$ & $\left.0,311^{* *}\right)$ & 0,04 & $\left.0,65^{(* *}\right)$ & $0,20\left(^{* *}\right)$ & $0,94\left(^{* *}\right)$ & $0,27\left(^{* *}\right)$ & $0,47\left(^{* *}\right)$ & $\left.0,411^{* *}\right)$ & $0,39\left(^{* *}\right)$ & $-0,06$ & $0,48\left({ }^{* *}\right)$ \\
\hline Histriônico & 0,06 & $-0,05$ & $0,48\left(^{* *}\right)$ & $0,15\left(^{* \star}\right)$ & $0,42\left(^{* \star}\right)$ & $\left.0,211^{* *}\right)$ & $0,13\left(^{*}\right)$ & $0,47\left(^{\star *}\right)$ & $0,15\left(^{* *}\right)$ & $0,68\left({ }^{* *}\right)$ & 0,10 & $0,24\left(^{\star *}\right)$ & $0,29\left(^{* *}\right)$ & $\left.0,72^{(* *}\right)$ & 0,10 \\
\hline Narcisista & $0,22\left(^{* *}\right)$ & $\left.0,211^{* *}\right)$ & $0,68\left(^{* *}\right)$ & $0,38\left(^{* *}\right)$ & $0,57\left(^{* *}\right)$ & $0,34\left(^{* *}\right)$ & $0,24\left(^{* *}\right)$ & $0,64\left(^{* *}\right)$ & $0,19\left(^{* *}\right)$ & $0,68\left(^{* *}\right)$ & $0,30\left(^{* *}\right)$ & $0,37\left(^{* *}\right)$ & $0,45\left(^{* *}\right)$ & $\left.0,611^{\star *}\right)$ & $0,25\left(^{* *}\right)$ \\
\hline Antissocial & $0,38\left(^{* *}\right)$ & $\left.0,311^{* *}\right)$ & $0,38\left(^{* *}\right)$ & $0,37\left(^{* *}\right)$ & $0,53\left(^{* *}\right)$ & 0,08 & $\left.0,311^{* *}\right)$ & $\left.0,88^{(* *}\right)$ & $0,27\left({ }^{* *}\right)$ & $0,47\left(^{* *}\right)$ & $\left.0,311^{* *}\right)$ & $0,47\left(^{* *}\right)$ & $0,47\left(^{* *}\right)$ & $0,26\left(^{* *}\right)$ & $0,35\left(^{* *}\right)$ \\
\hline Sádico & $0,46\left(^{* \star}\right)$ & $0,38\left(^{* *}\right)$ & $0,39\left(^{* *}\right)$ & $0,47\left(^{* *}\right)$ & $0,87\left(^{* \star}\right)$ & $0,15\left(^{* *}\right)$ & $0,42\left(^{* *}\right)$ & $0,60\left(^{* *}\right)$ & $0,24\left(^{* *}\right)$ & $\left.0,511^{* *}\right)$ & $\left.0,40^{(*}\right)$ & $0,40\left(^{* *}\right)$ & $0,79\left(^{* *}\right)$ & $\left.0,18^{(* *}\right)$ & $\left.0,422^{* *}\right)$ \\
\hline Compulsivo & $0,16\left(^{* *}\right)$ & $0,27\left(^{* *}\right)$ & $0,34\left(^{* *}\right)$ & $0,33\left({ }^{* \star}\right)$ & 0,08 & $0,94\left(^{* \star}\right)$ & $0,22\left(^{* *}\right)$ & $0,18\left(^{* *}\right)$ & 0,05 & $\left.0,211^{\star *}\right)$ & $0,23\left({ }^{* *}\right)$ & 0,09 & $0,17\left(^{* *}\right)$ & $0,24\left(^{* *}\right)$ & 0,06 \\
\hline Negativista & $0,68\left(^{* *}\right)$ & $0,64\left(^{* *}\right)$ & $\left.0,611^{* *}\right)$ & $0,62\left(^{* *}\right)$ & $\left.0,511^{* \star}\right)$ & $0,25\left(^{* *}\right)$ & $0,72\left(^{* *}\right)$ & $\left.0,411^{* \star}\right)$ & $0,47\left({ }^{* *}\right)$ & $0,58\left(^{* *}\right)$ & $0,84\left(^{* \star}\right)$ & $0,47\left(^{* *}\right)$ & $0,60\left(^{* *}\right)$ & 0,06 & $0,60\left({ }^{* *}\right)$ \\
\hline Masoquista & $0,77\left(^{* *}\right)$ & $0,64\left(^{* *}\right)$ & $0,44\left({ }^{* *}\right)$ & $0,47\left(^{* *}\right)$ & $0,42\left(^{* *}\right)$ & 0,10 & $\left.0,75^{(* *}\right)$ & $0,26\left(^{* *}\right)$ & $\left.0,50^{(* *}\right)$ & $0,29\left(^{* *}\right)$ & $0,63\left(^{* *}\right)$ & $0,52\left(^{* *}\right)$ & $0,52\left(^{* *}\right)$ & $-0,10$ & $0,72\left(^{* *}\right)$ \\
\hline Paranoide & $0,55\left(^{\star *}\right)$ & $0,55\left(^{* *}\right)$ & $0,49\left(^{* *}\right)$ & $0,94\left(^{* *}\right)$ & $\left.0,411^{* *}\right)$ & $\left.0,411^{* *}\right)$ & $0,63\left(^{* *}\right)$ & $0,37\left(^{* *}\right)$ & $0,32\left(^{* *}\right)$ & $\left.0,411^{* *}\right)$ & $0,63\left(^{* *}\right)$ & $0,46\left(^{* *}\right)$ & $0,53\left(^{* \star}\right)$ & 0,06 & $\left.0,411^{* *}\right)$ \\
\hline Esquizotípico & $0,69\left(^{* *}\right)$ & $0,63\left({ }^{* *}\right)$ & $0,49\left(^{* *}\right)$ & $0,50\left(^{* *}\right)$ & $0,42\left(^{* *}\right)$ & $0,15\left(^{* *}\right)$ & $0,62\left(^{* *}\right)$ & $0,42\left(^{* *}\right)$ & $0,44\left(^{* *}\right)$ & $0,39\left(^{* *}\right)$ & $0,53\left(^{* *}\right)$ & $0,90\left(^{* *}\right)$ & $0,83\left({ }^{* *}\right)$ & $0,06\left(^{* *}\right)$ & $0,49\left(^{* *}\right)$ \\
\hline Borderline & $0,75\left(^{\star *}\right)$ & $0,56\left(^{* *}\right)$ & $0,76\left(^{* *}\right)$ & $\left.0,50^{(* *}\right)$ & $0,45\left(^{* *}\right)$ & $0,18\left(^{* *}\right)$ & $0,65\left(^{* *}\right)$ & $0,42\left({ }^{* *}\right)$ & $0,57\left(^{* *}\right)$ & $0,41\left(^{* *}\right)$ & $0,57\left(^{* *}\right)$ & $0,58\left(^{* *}\right)$ & $0,56\left(^{* *}\right)$ & 0,06 & $\left.0,50{ }^{* *}\right)$ \\
\hline
\end{tabular}

$\left(^{*}\right)$ Estatisticamente significativos ao nível de 0,05 $\left(^{* \star}\right)$ Estatisticamente significativos ao nível de 0,01

Nota. As correlações com as maiores magnitudes foram marcadas em negrito. Para diminuição da possibilidade do erro Tipo I, por meio do software estatístico G*Power versão 3.1.5, calculou-se a magnitude mínima das correlações para obtenção de um poder estatístico (1 - $\beta$ ) de 0,80 (Faul, Erdfelder, Buchner, \& Lang, 2009 ; Faul, Erdfelder, Lang, \& Buchner, 2007). A partir disso, considera-se o coeficiente 0,14 como significativo $(p=<0,05)$ e com poder estatístico minimamente adequado. 
discriminação (por exclusão de itens que apresentaram overlapping), e a representabilidade (pela inserção de itens) dos fatores. Com base nesses critérios e pautado na pertinência teórica das mudanças dos itens nos fatores, procedeu-se às seguintes alterações: os itens $89 \mathrm{e}$ 15 foram excluídos do fator 1; os itens 1,5 e 78 foram excluídos do fator 2; os itens 66 e 81 foram excluídos do fator 9; o item 67 foi incluído no fator 10; o item 41 foi inserido no fator 11; e, o item 72 foi excluído dos fatores 12 e 13 (que foram unidos, como descrito anteriormente). Por fim, como não foram obtidos fatores empíricos para avaliação dos transtornos da personalidade evitativo e narcisista, foram utilizados os conjuntos de itens hipotetizados teoricamente par esses construtos. Como resultado das análises previamente descritas, foi obtida a versão final do IDTP. As 14 escalas do IDTP são apresentadas na Tabela 5.

A versão final do instrumento ficou composta por 75 itens, sendo que alguns itens foram computados em mais de uma escala (por exemplo, o item 48 corresponde às escalas Borderline e Evitativo). A escala com menor número de itens (5 itens) foi a Escala Histriônico, e com o maior número de itens foi a Escala Depressivo (9 itens). Os coeficientes alfa variaram entre $0,65 \mathrm{e}$ 0,89 , sendo que a Escala Narcisista foi a única que apresentou índice de fidedignidade inferior a 0,71 . Na última coluna da Tabela 5 são apresentadas as porcentagens de itens que eram esperados teoricamente em cada fator. Verificou-se que a maior parte das escalas

Tabela 4

Coeficientes de Correlação de Pearson entre os fatores empíricos interpretáveis e não interpretáveis

\begin{tabular}{|c|c|c|c|c|c|c|c|c|}
\hline Fatores & F16 & F17 & F18 & F19 & F20 & F21 & F22 & F23 \\
\hline F1 & $0,27\left({ }^{* *}\right)$ & $0,40\left(^{* *}\right)$ & $0,48(* *)$ & $0,38\left({ }^{* *}\right)$ & $0,44\left({ }^{* *}\right)$ & $0,33\left({ }^{* *}\right)$ & $0,46\left({ }^{* *}\right)$ & 0,10 \\
\hline F2 & $0,26\left(^{* *}\right)$ & $\left.0,311^{* *}\right)$ & $0,39\left(^{* *}\right)$ & $\left.0,311^{* *}\right)$ & $0,37\left(^{* *}\right)$ & $\left.0,511^{* \star}\right)$ & $0,42\left(^{* \star}\right)$ & 0,03 \\
\hline F3 & $0,38\left(^{* *}\right)$ & $0,18\left(^{* *}\right)$ & $0,41\left(^{* *}\right)$ & $0,54(* *)$ & $0,27\left({ }^{* *}\right)$ & $0,29\left({ }^{* *}\right)$ & $0,44\left(^{* *}\right)$ & $0,32\left({ }^{* *}\right)$ \\
\hline F4 & $0,33\left({ }^{* *}\right)$ & $0,28\left({ }^{* *}\right)$ & $0,55\left(^{* *}\right)$ & $0,33\left({ }^{* *}\right)$ & $0,52\left({ }^{* *}\right)$ & $0,34\left({ }^{* *}\right)$ & $0,44\left({ }^{* *}\right)$ & $0,17\left(^{* *}\right)$ \\
\hline F5 & $0,64(* *)$ & $0,29\left({ }^{* *}\right)$ & $0,42(* *)$ & $0,34(* *)$ & $0,29\left({ }^{* *}\right)$ & $0,21\left({ }^{* *}\right)$ & $0,33(* *)$ & $0,40\left({ }^{* *}\right)$ \\
\hline F6 & $0,17\left({ }^{* *}\right)$ & 0,04 & $0,13\left(^{*}\right)$ & $0,21\left(^{* *}\right)$ & 0,04 & $0,32\left(^{* *}\right)$ & $0,21\left(^{* *}\right)$ & $0,13\left(^{*}\right)$ \\
\hline F7 & $0,29\left({ }^{* *}\right)$ & $0,33\left({ }^{* *}\right)$ & $0,42\left({ }^{* *}\right)$ & $0,44\left({ }^{* *}\right)$ & $0,38\left({ }^{* *}\right)$ & $0,33\left({ }^{* *}\right)$ & $0,50\left({ }^{* *}\right)$ & $0,13\left(^{*}\right)$ \\
\hline F8 & $0,42\left({ }^{* *}\right)$ & $0,33\left({ }^{* *}\right)$ & $0,33\left({ }^{* *}\right)$ & $0,31\left({ }^{* *}\right)$ & $0,19\left({ }^{* *}\right)$ & $0,27\left({ }^{* *}\right)$ & $0,24\left({ }^{* *}\right)$ & $0,66\left(^{* *}\right)$ \\
\hline F9 & $0,18\left(^{* *}\right)$ & $0,22(* *)$ & $0,37\left({ }^{* *}\right)$ & $0,36\left({ }^{* *}\right)$ & $0,27\left({ }^{* *}\right)$ & $0,14\left({ }^{* *}\right)$ & $0,30\left(^{* *}\right)$ & $0,12\left(^{*}\right)$ \\
\hline F10 & $0,47\left({ }^{* *}\right)$ & $0,21\left({ }^{* *}\right)$ & $0,36\left({ }^{* *}\right)$ & $0,33\left({ }^{* *}\right)$ & $0,23\left({ }^{* *}\right)$ & $0,28\left({ }^{* *}\right)$ & $0,38\left({ }^{* *}\right)$ & $\left.0,466^{* *}\right)$ \\
\hline F11 & $0,28\left({ }^{* *}\right)$ & $0,33\left({ }^{* *}\right)$ & $0,54\left({ }^{* *}\right)$ & $0,32\left({ }^{* *}\right)$ & $0,55\left(^{* *}\right)$ & $0,33\left({ }^{* *}\right)$ & $0,49\left({ }^{* *}\right)$ & 0,05 \\
\hline F12 & $0,30\left(^{* *}\right)$ & $0,34\left({ }^{* *}\right)$ & $0,29(* *)$ & $0,38(* *)$ & $0,24\left({ }^{* *}\right)$ & $0,22\left({ }^{* *}\right)$ & $0,38(* *)$ & $0,28\left({ }^{* *}\right)$ \\
\hline F13 & $0,34\left({ }^{* *}\right)$ & $0,38\left(^{* *}\right)$ & $0,36\left(^{* *}\right)$ & $0,38\left(^{* *}\right)$ & $0,29\left({ }^{* *}\right)$ & $0,31\left({ }^{* *}\right)$ & $0,38\left({ }^{* *}\right)$ & $0,30(* *)$ \\
\hline F14 & $0,18\left(^{* *}\right)$ & 0,06 & 0,02 & $0,10\left(^{*}\right)$ & $-0,04$ & $0,15\left(^{* *}\right)$ & 0,03 & $0,57\left({ }^{* *}\right)$ \\
\hline F15 & $0,26\left(^{* *}\right)$ & $0,32\left({ }^{* *}\right)$ & $0,43\left({ }^{* *}\right)$ & $0,30\left(^{* *}\right)$ & $0,41\left({ }^{* *}\right)$ & $0,25\left(^{* *}\right)$ & $0,37\left({ }^{* *}\right)$ & $0,16\left({ }^{* *}\right)$ \\
\hline
\end{tabular}

(*) Estatisticamente significativos ao nivel de $0,05\left(^{* *}\right)$ Estatisticamente significativos ao nível de 0,01

Nota. As correlações com as maiores magnitudes foram marcadas em negrito. Para diminuição da possibilidade do erro Tipo I, por meio do software estatístico G*Power versão 3.1.5, calculou-se a magnitude mínima das correlações para obtenção de um poder estatístico (1 - $\beta$ ) de 0,80 (Faul et al., 2009 ; Faul et al., 2007). A partir disso, considera-se o coeficiente 0,14 como significativo $(p=<0,05)$ e com poder estatístico minimamente adequado.

apresentou porcentagem superior a $50 \%$, com exceção das escalas Borderline, Masoquista e Esquizoide. $\mathrm{Na}$ continuidade, a Tabela 6 apresenta as estatísticas descritivas referentes aos escores fatoriais, resultados da aplicação do instrumento.

Verifica-se na Tabela 6 que, em geral, foram dadas respostas às escalas, que contemplaram o mínimo (1) e o máximo (4) na escala Likert, com exceção às escalas Borderline, Masoquista, Dependente, Negativista, e Narcisista. A média das respostas na maioria dos fatores foi abaixo do ponto central $(2,5)$, sendo a mais bai- xa 1,52 (Escala Sádico) e a mais alta 2,76 (Escala Compulsivo). E, o desvio padrão pode ser considerado baixo em todos os fatores, variando entre 0,46 (Escala Negativista) e 0,67 (escalas Compulsivo e Esquizotípico), sugerindo uma concentração de respostas pelos sujeitos que responderam o instrumento. Por último, visando à verificação da possibilidade de reagrupamento dos quatorze fatores primários (somente as escalas de transtornos da personalidade) em fatores de segunda ordem, procedeu-se a uma análise por componentes principais de segunda ordem (por componentes 
Tabela 5

Itens e Coeficiente de Fidedignidade das Escalas do IDTP

\begin{tabular}{lccc}
\hline Escalas & Itens & Coef. a & $\begin{array}{c}\text { \% Itens Esperados } \\
\text { Teoricamente }\end{array}$ \\
\hline Depressivo (Escala I) & $49,18,80,6,46,99,73,34,92$ & 0,89 & $55,5 \%$ \\
Esquizoide (Escala II) & $16,62,31,76,93,95,79,63$ & 0,86 & $50 \%$ \\
Borderline (Escala III) & $51,61,48,37,52,17,77,21$ & 0,79 & $25 \%$ \\
Paranoide (Escala IV) & $59,28,43,90,13,26$ & 0,77 & $83,3 \%$ \\
Sádico (Escala V) & $9,54,70,67,21,24$ & 0,76 & $66,6 \%$ \\
Compulsivo (Escala VI) & $13,25,4,71,56,40$ & 0,77 & $83,3 \%$ \\
Masoquista (Escala VII) & $97,96,32,98,81,58,41,27$ & 0,81 & $37,5 \%$ \\
Antissocial (Escala VIII) & $38,84,69,85,83,53$ & 0,73 & $66,6 \%$ \\
Dependente (Escala IX) & $7,19,35,50,66,81$ & 0,77 & $100 \%$ \\
Histriônico (Escala X) & $82,2,68,72,67$ & 0,71 & $60 \%$ \\
Negativista (Escala XI) & $26,64,88,57,94,41$ & 0,74 & $66,6 \%$ \\
Esquizotípico (Escala XII) & $45,29,60,14,91,72$ & 0,76 & $83,3 \%$ \\
Evitativo (Escala XIII) & $63,5,79,48,17,32,94$ & 0,81 & $100 \%$ \\
Narcisista (Escala XIV) & $37,52,21,83,3,68$ & 0,65 & $100 \%$ \\
\hline
\end{tabular}

principais e rotação oblíqua - directed oblimin). Foram obtidos três fatores com eigenvalue acima de 1,0, capazes de explicar $71,9 \%$ da variância total. As cargas fatoriais da matriz rotada são apresentadas na Tabela 7 .

Pode-se notar que, as escalas Evitativo, Depressivo, Masoquista, Esquizoide, Negativista, Dependente, Paranoide e Esquizotípico se agruparam prioritariamente no primeiro fator de segunda ordem (FSO1). No segun- do fator (FSO2) foram agrupadas as escalas Narcisista, Histriônico, Antissocial e Sádico. E, o último fator, fator 3 (FSO3), é representado prioritariamente pela Escala Compulsivo. A Escala Borderline apresentou cargas similares nos três fatores, contudo, prioritariamente no fator FSO1. Ainda, no que se refere à fidedignidade desses fatores, os coeficientes variaram entre 0,77 e 0,92 .

Tabela 6

Estatísticas Descritivas das escalas da versão final do instrumento

\begin{tabular}{lccccc}
\hline Fatores & N & Mínimo & Máximo & Média & Desvio Padrão \\
\hline Depressivo (Escala I) & 347 & 1,0 & 4,0 & 1,54 & 0,64 \\
Esquizoide (Escala II) & 345 & 1,0 & 4,0 & 1,55 & 0,53 \\
Borderline (Escala III) & 345 & 1,14 & 4,0 & 2,46 & 0,61 \\
Paranoide (Escala IV) & 346 & 1,0 & 4,0 & 1,84 & 0,57 \\
Sádico (Escala V) & 347 & 1,0 & 4,0 & 1,52 & 0,50 \\
Compulsivo (Escala VI) & 348 & 1,0 & 4,0 & 2,76 & 0,67 \\
Masoquista (Escala VII) & 347 & 1,0 & 3,6 & 1,59 & 0,56 \\
Antissocial (Escala VIII) & 346 & 1,0 & 4,0 & 1,72 & 0,55 \\
Dependente (Escala IX) & 347 & 1,0 & 3,5 & 1,56 & 0,53 \\
Histriônico (Escala X) & 346 & 1,0 & 4,0 & 1,73 & 0,59 \\
Negativista (Escala XI) & 345 & 1,0 & 3,33 & 1,46 & 0,46 \\
Esquizotípico (Escala XII) & 342 & 1,0 & 4,0 & 1,79 & 0,67 \\
Evitativo (Escala XIII) & 347 & 1,0 & 4,0 & 1,79 & 0,60 \\
Narcisista (Escala XIV) & 346 & 1,13 & 3,9 & 2,18 & 0,54 \\
\hline
\end{tabular}




\section{DISCUSSÃO}

A versão do IDTP, obtida na primeira etapa deste estudo, e utilizada na fase de aplicação da pesquisa, foi composta por 92 itens, distribuídos entre 14 escalas distintas. Quando comparado com outros instrumentos com objetivos similares, como o MCMI-III e o MMPI2, que contêm, respectivamente, 175 itens e 567 itens, o IDTP pode ser considerado um instrumento breve, o que é desejável para instrumentos para o contexto clínico. Entretanto, ao menos duas distinções entre o IDTP e os instrumentos já existentes devem ser apontadas.

A primeira delas concerne ao fato de que a avaliação de transtornos da personalidade realizada pelo IDTP tem fins de triagem e não de diagnóstico, como os dois instrumentos citados. E, a segunda, respeita à abrangência desses instrumentos. Por um lado, o IDTP busca uma avaliação dos transtornos da personalidade com base na teoria de Millon (Millon \& cols., 2004), portan-

Tabela 7

Cargas fatoriais da matriz rotada de segunda ordem e coeficiente alfa de Cronbach

\begin{tabular}{lccc}
\hline Fatores & FSO1 & FSO2 & FSO3 \\
\hline Evitativo (Escala XIII) & 0,916 & & \\
Depressivo (Escala I) & 0,882 & & \\
Masoquista (Escala VII) & 0,866 & & \\
Esquizoide (Escala II) & 0,810 & & \\
Negativista (Escala XI) & 0,791 & & \\
Dependente (Escala IX) & 0,682 & & \\
Paranoide (Escala IV) & 0,531 & & \\
Esquizotípico (Escala XII) & 0,523 & 0,378 & \\
Borderline (Escala III) & 0,449 & 0,301 & 0,338 \\
Narcisista (Escala XIV) & & 0,932 & \\
Histriônico (Escala X) & & 0,836 & \\
Antissocial (Escala VIII) & & 0,828 & \\
Sádico (Escala V) & & 0,750 & \\
Compulsivo (Escala VI) & & & 0,921 \\
\hline Coeficiente Alfa & 0,92 & 0,86 & $0,77^{*}$ \\
\hline
\end{tabular}

"Uma vez que o fator $\mathrm{FSO} 3$ foi composto por apenas uma variável, 0 cálculo do coeficiente alfa foi baseado nos itens que compõem a escala Compulsivo.

to, refere-se a uma avaliação de 14 diferentes construtos. Por outro, tanto o MCMI-III quanto o MMPI-2 buscam, além da avaliação de diversos transtornos da personalidade, também a avaliação dos transtornos des- critos no Eixo I do DSM-IV-TR (APA, 2003). Essas considerações resultam diretamente em uma diminuição do número de itens do instrumento presentemente desenvolvido, o que é desejado para aplicações no âmbito da clínica (Millon \& Davis, 1996). Não obstante, deve-se considerar que atualmente não há instrumento aprovado pelo Conselho Federal de Psicologia (CFP) para triagem de funcionamentos patológicos da personalidade (vide o link satepsi.cfp.org.br/).

Para verificação da estrutura do instrumento desenvolvido, procedeu-se tanto à análise por componentes principais de primeira ordem quanto à análise de segunda ordem, esta última com o objetivo de agrupar as dimensões encontradas anteriormente. No que concerne à análise de primeira ordem, esperava-se que fossem encontrados quatorze fatores distintos que correspondessem aos transtornos da personalidade considerados na teoria de Millon. Contudo, dos 15 fatores interpretáveis (de 23 fatores obtidos), puderam ser identificados somente 12 fatores correspondentes aos construtos esperados. Por isso, foram utilizadas duas escalas teoricamente esperadas para a versão final do instrumento: Evitativo e Narcisista. Vale ressaltar também que a composição final de cada uma das escalas, por vezes, agrupou itens representando mais de um transtorno (por exemplo, itens relacionados ao transtorno da personalidade evitativo na escala Esquizoide). Contudo, em todos os casos em que isso ocorreu, a análise do conteúdo dos itens demonstrou pertinência da manutenção do item na escala.

Ainda no que concerne à estrutura interna encontrada, era esperado que fossem encontradas empiricamente quatorze escalas referentes aos transtornos da personalidade propostos por Millon. Contudo, é explícita na literatura a dificuldade em se encontrar empiricamente o que é proposto teoricamente, sobretudo no que concerne a modelos complexos como o de Millon (Millon \& Davis, 1996; Millon, 2011). Millon argumenta que dificilmente sua teoria pode ser evidenciada como um todo por meio de procedimentos como a análise por componentes principais, por se tratar de um modelo complexo para o entendimento e avaliação da personalidade e seus transtornos (Strack \& Millon, 2007).

Especificamente à respeito a uma das escalas do instrumento, Escala Narcisista, estudos anteriores apontam para baixos índices de fidedignidade dessa escala no MCMI-III, variando entre 0,55 e 0,67 (Millon et al., 1994; Millon \& Davis, 1996; Rossi et al., 2007; 
Rossi et al., 2003). Esses dados, semelhantes ao dado presentemente evidenciado $(\alpha=0,65)$, sugerem dificuldades na avaliação do transtorno da personalidade narcisista, seja por inadequações do instrumento, ou por dificuldades inerentes ao próprio construto (Millon et al., 2004). Além disso, é proposto na literatura que as dificuldades na avaliação de alguns transtornos da personalidade, como o Narcisista (e também o Compulsivo), ocorrem devido à diferenciação das características dos itens formulados para esses transtornos em relação aos outros, já que são frequentemente itens com características mais positivas (Strack \& Millon, 2007; Choca, 2004).

Na continuidade, após as análises para estabelecimento da estrutura interna, a versão final do IDTP ficou composta de 75 itens distribuídos em 14 escalas. A Tabela 8 apresenta uma comparação entre os índices de fidedignidade presentemente encontrados com o IDTP e os dados encontrados em estudos anteriores com o MCMI-III.

Como pode ser observado na tabela apresentada, os índices de fidedignidade das escalas para avaliação de transtornos da personalidade do IDTP e das escalas do MCMI-III em diferentes estudos são similares. Esses dados podem ser considerados como favoráveis para o IDTP, dado o número reduzido de itens em relação ao MCMI-III, e também as limitações da amostra deste estudo, já que a maior parte dos participantes não tem diagnóstico psiquiátrico conhecido.
Já na análise de segunda ordem, realizada a partir dos fatores utilizados para a versão final do IDTP, foram encontrados 3 dimensões. Resultados semelhantes com as escalas para avaliação dos transtornos da personalidade do MCMI-III foram apresentados no estudo realizado por Dyce et al. (1997). Entre outros resultados, foi encontrada uma solução fatorial de 3 fatores, sendo o fator 1 composto pelas escalas Esquizoide, Evitativo, Depressivo, Dependente, Histriônico, Negativista, Masoquista, Esquizotípico e Borderline; o fator 2 pelas escalas Narcisista, Antissocial, Sádico e Paranoide; e o fator 3 pela Escala Compulsivo.

Basicamente, as diferenças entre os dados presentemente encontrados e os resultados evidenciados por Dyce et al. (1997), são, o fator 1 deste estudo engloba a Escala Paranoide; o fator 1 do estudo de Dyce et al. engloba a Escala Histriônico; o fator 2 deste estudo engloba as escalas Histriônico e Borderline; e, o fator 2 do estudo de Dyce e cols. engloba a Escala Paranoide. Esses dados sustentam a consistência da estrutura fatorial encontrada nesta pesquisa. Futuros estudos devem buscar replicar o agrupamento das escalas em 3 dimensões, buscando compreender com mais profundidade o significado do modo com que as mesmas se aglomeraram. Ainda assim, é possível notar uma tendência para agrupar funcionamentos mais passivos (Millon, 2011) ou internalizantes (Krueger et al., 2011) no fator 1; mais ativos e agressivos ou externalizantes no fator 2; e um único funcionamento isolado no último fator, o que pode indicar alguma peculiaridade da própria escala de

Tabela 8

Coeficientes de fidedignidade do IDTP e do MCMI-III

\begin{tabular}{lcccc}
\hline Escalas & $\begin{array}{c}\text { Presente } \\
\text { estudo }\end{array}$ & $\begin{array}{c}\text { Millon et al., } \\
1994\end{array}$ & $\begin{array}{c}\text { Rossi et al., } \\
\mathbf{2 0 0 7}\end{array}$ & $\begin{array}{c}\text { Rossi et al., } \\
\mathbf{2 0 0 3}\end{array}$ \\
\hline Depressivo & 0,89 & 0,89 & 0,88 & 0,87 \\
Esquizoide & 0,86 & 0,81 & 0,72 & 0,82 \\
Borderline & 0,79 & 0,85 & 0,82 & 0,80 \\
Paranoide & 0,77 & 0,84 & 0,80 & 0,76 \\
Sádico & 0,76 & 0,79 & 0,76 & 0,65 \\
Compulsivo & 0,77 & 0,66 & 0,68 & 0,70 \\
Masoquista & 0,81 & 0,87 & 0,84 & 0,75 \\
Antissocial & 0,73 & 0,77 & 0,72 & 0,69 \\
Dependente & 0,77 & 0,85 & 0,82 & 0,81 \\
Histriônico & 0,71 & 0,81 & 0,80 & 0,70 \\
Negativista & 0,74 & 0,83 & 0,78 & 0,72 \\
Esquizotípico & 0,76 & 0,85 & 0,84 & 0,80 \\
Evitativo & 0,81 & 0,89 & 0,86 & 0,82 \\
Narcisista & 0,65 & 0,67 & 0,67 & 0,55 \\
\hline
\end{tabular}


avaliação (a literatura ressalta dificuldade na avaliação desse funcionamento).

Craig e Bivens (1998) também encontraram uma solução composta por 3 fatores para as 14 escalas do MCMI-III. O primeiro fator encontrado no estudo agrupou as escalas Esquizoide, Evitativo, Depressivo, Dependente, Histriônico (carga negativa), Narcisista (carga negativa), Compulsivo (carga negativa), Masoquista e Borderline; o segundo fator consistiu das escalas Negativista, Esquizotípico e Paranoide; e, o terceiro e último fator agrupou as escalas Antissocial, Sádico, Compulsivo (carga negativa) e Borderline. Diferente do estudo apresentado anteriormente, as discrepâncias entre os dados encontrados no presente estudo e no estudo de Craig e Bivens são mais evidentes. Ainda assim, o primeiro fator de ambos os estudos compartilham 6 escalas (Esquizoide, Evitativo, Depressivo, Dependente, Masoquista e Borderline). Cabe apontar que a Escala Compulsivo, que neste estudo compôs um fator isolado, obteve carga negativa em dois fatores $(1$ e 3$)$ no estudo de Craig e Bivens, o que parece ser mais uma evidência de dificuldades na avaliação de escalas de transtornos da personalidade que contenham atributos positivos ou socialmente desejados (Strack \& Millon, 2007).

Em síntese, no que se refere às estruturas fatoriais evidenciadas, neste estudo e em estudos anteriores, como apontam Dyce et al. (1997) e Strack e Millon (2007), não é esperado que as complexidades da teoria de Millon sejam completamente encontradas e validadas por meio da análise por componentes principais. Contudo, é importante que as soluções fatoriais evidenciadas nos diferentes estudos apresentem dados persistentes ao longo do tempo. Desse modo, pode-se considerar que os dados encontrados apontam para uma persistência de uma estrutura fatorial similar, mas ainda assim, com algumas diferenciações importantes.

\section{CONSIDERAÇÕES FINAIS}

O presente estudo teve como objetivo a construção e validação de um instrumento, com base na teoria de Millon e nos instrumentos derivados dela, para avaliação de transtornos da personalidade. Em geral, os dados obtidos, tanto no que se refere à construção do instrumento quanto às evidências de validade e fidedignidade, foram satisfatórios. No que concerne à estrutura interna do IDTP, a partir da análise por componentes principais e análises subsequentes, lapidou-se um instrumento para avaliação dos transtornos da personalidade composto por 75 itens, distribuídos em 14 escalas, que devem ser respondidos em uma escala Likert de 4 pontos.

Como já apontado em publicações nacionais (Carvalho, Bartholomeu \& Silva, 2010), há uma evidente lacuna na área de avaliação de funcionamentos patológicos e transtornos da personalidade no Brasil. Nesse contexto, a ferramenta aqui apresentada configura-se como uma das possibilidade de uso para a área clínica, possibilitando ao profissional uma triagem inicial acerca de funcionamentos patológicos da personalidade dos pacientes.

Apesar das adequações psicométricas evidenciadas para o instrumento, algumas limitações devem ser apontadas neste estudo. A primeira delas refere-se a uma limitação frequente nos estudos na área de psicologia, que é a não aleatoriedade e representatividade amostral dos participantes do estudo, isto é, a amostra do presente estudo não foi aleatória e randomizada, mas optou-se pela amostra por conveniência. Ainda sobre a amostra, e configurando uma segunda limitação deste estudo, dado o objetivo de avaliação do instrumento construído, seria importante que a amostra de pacientes psiquiátricos tivesse sido mais representativa, isto é, com maior número de participantes nesse grupo.

Ao lado disso, também podem ser apontadas sugestões para futuros estudos que tenham como pretensão dar continuidade para os achados com o IDTP. De maneira mais global, propõe-se que este estudo seja replicado, de maneira a verificar se a estrutura fatorial presentemente evidenciada se repete em outras amostras. Ainda, é de grande importância que, além da validade fatorial do instrumento, sejam investigadas evidências de validade por meio de critérios externos. De outro modo, mais especificamente, seria interessante que novos itens para as escalas Evitativo e Narcisista fossem construídos, de modo que essas escalas fossem evidenciadas empiricamente. Nesse mesmo sentido, novos itens para a escala Narcisista poderiam ser desenvolvidos, na tentativa de melhorar o coeficiente de fidedignidade dessa escala.

\section{REFERÊNCIAS}

Alchieri, J. C. (2004). Modelo dos Estilos de Personalidade de Millon: Adaptação do Inventário Millon de Estilos de Personalidade. Tese de doutorado não publicada, Programa de Pós-Graduação em Psicologia, Universidade Federal do Rio Grande do Sul, 180 pp.

American Psychiatry Association. (2013). Diagnostic and Statistical Manual of Mental Disorders 5. Washington: American Psychiatry Association. 
American Psychological Association. (2003). Manual Diagnóstico e Estatístico de Transtornos Mentais DSM-IV-TR ( $4^{\mathrm{a}}$ ed.). Porto Alegre: Artmed.

Beck, A. T.; Freeman, A., \& Davis, D. T. (2005). Terapia cognitiva dos transtornos da personalidade. Porto Alegre: Artmed.

Caballo. V. E. (2007). Manual de Transtornos de Personalidade. São Paulo: Santos.

Carlat, D. J. (2006). Entrevista Psiquiátrica. 2a edição. Porto Alegre: Artmed.

Carvalho, L. F., \& Primi, R. (2013). Perspectivas em psicologia dos transtornos da personalidade. São Paulo: Casa do Psicólogo. 516 páginas.

Carvalho, L. F.; Bartholomeu, D.; \& Silva, M. C. R. (2010). Instrumentos para avaliação dos transtornos da personalidade no Brasil. Avaliação Psicológica, 9(2), 289-298.

Carvallho, L.F., \& Ambiel, R.A.M. (2010). Considerações gerais acerca da proposta de Teofrasto para o estudo da personalidade. Revista Psicologia e Saúde, 2 (2), 76-80.

Choca, J. P. (2004). Interpretative Guide for the Millon Clinical Multiaxial Inventory III. Washington, DC: American Psychological Association.

Craig, R. J. (1999). Overview and current status of the Millon Clinical Multiaxial Inventory. Journal of Personality Assessment, 72 (3), 390-406.

Craig, R. J., \& Bivens, A. (1998). Factor structure of the MCMI-III. Journal of Personality Assessment, 70, 190-196.

Craig, R. J., \& Olson, R. E. (2001). Adjectival Descriptions of Personality Disorders: A Convergent Validity Study of the MCMI-III. Journal of Personality Assessment, 77 (2), 259-271.

Cunha, J. A. (2001). Manual da versão em português das Escalas Beck. São Paulo: Casa do Psicólogo.

Davis, R. D. (1999). Millon: Essentials of his science, theory, classification, assessment, and therapy. Journal of Personality Assessment, 72 (3), 330-352.

Del Porto, J. A. (1999). Conceito e diagnóstico. Revista Brasileira de Psiquiatria, 21, 6-11.

Dyce, J. A., O'Connor, B. P., Parkins, S., \& Janzen, H. (1997). Correlational structure of the MCMI-III personality disorder scales and comparison with other data sets. Journal of Personality Assessment, 69, (3), 568-582.

Faul, F., Erdfelder, E., Buchner, A., \& Lang, A.-G. (2009). Statistical power analyses using $\mathrm{G}^{*}$ Power 3.1: Tests for correlation and regression analyses. Behavior Research Methods, 41, 1149-1160.

Faul, F., Erdfelder, E., Lang, A.-G., \& Buchner, A. (2007). G*Power 3: A flexible statistical power analysis program for the social, behavioral, and biomedical sciences. Behavior Research Methods, 39, 175-191.

Gunther, H. (1999). Como elaborar um questionário. Em: L. Pasquali, (Org), Instrumentos psicológicos: manual prático de elaboração (pp. 231-258) Brasília: LabPAM/IBAPP.

Haddy, C., Strack, S. \& Choca, J. P. (2005). Linking Persona- lity Disorders and Clinical Syndromes on the MCMI-III. Journal of Personality Assessment, 84 (2), 193-204.

Handler, L. \& Meyer, G. J. (1997). The importance of teaching and learning personality assessment. In: Handler, L., \& Hilsenroth, M. (eds), Teaching and learning personality assessment (pp. 3-30) New Jersey: Lawrence Erlbaum Associates.

John, O. P., Robins, R. W., \& Pervin, L. A. (2008). Handbook of personality: theory and research. Third edition. New York: The Guilford Press.

Krueger, R. F.; Eaton, N. R.; Derringer, J.; Markon, K. E.; Watson, D.; \& Skodol, A. E. (2011). Personality in DSM5: helping delineate personality disorder content and framing the metastructure. Journal of Personality Assessment, 93(4), 325-331.

Loureiro, S. R.C (2000). Transtornos de Personalidade e avaliação psicodiagnóstica. Em: F. F. Sisto, E. T. B. Sbardelini, \& R. Primi, (Orgs), Contextos e questões da avaliação psicológica (pp. 51-61) São Paulo: Casa do Psicólogo.

Millon, T. \& Davis, R. D. (1996). Disorders of Personality DSM-IV and Beyond. New Jersey: Wiley.

Millon, T. (1986). A Theoretical Derivation of Pathological Personalities. In: Millon, T., \& Klerman, G.L. (eds.), Contemporary Directions in Psychopathology: Toward the DSM-IV (pp. 639-670) Nova York: Guildford.

Millon, T. (2011). Disorders of Personality: introducing a $D S M / I C D$ spectrum from normal to abnormal. New Jersey: Wiley.

Millon, T. Millon, C. M., Meagher, S. Grossman, S. \& Ramanath, R. (2004). Personality Disorders in Modern Life. New Jersey: Wiley, 2004.

Millon, T., \& Grossman, S. (2007). Moderating severe personality disorders. John Wiley \& Sons Inc., New Jersey.

Millon, T., \& Grossman, S. (2007). Overcoming resistant personality disorders. John Wiley \& Sons Inc., New Jersey.

Millon, T., Grossman, S., \& Tringone, R. (2010). The Millon Personality Spectrometer: a tool for personality spectrum analyses, diagnoses, and treatments. Em: Millon, T., Krueger, R. F., \& Simonsen. (Orgs.), Contemporary directions in psychopathology: scientific foundations of the DSM-V and ICD-11 (391-418) The Guilford Press, New York.

Millon, T., Millon, C. M. \& R. D., Davis. (1994). MCMI-III Manual. Minneapolis: Dicandrien.

Morana, H. C. P. (2003). Identificação do ponto de corte para a escala PCL-R (Psychopathy Checklist Revised) em população forense brasileira: caracterização de dois subtipos da personalidade; transtorno global e parcial. Tese de doutorado não publicada, Programa de pós-graduação em Medicina, Universidade de São Paulo, 178pp.

Mullins-Sweatt, S. N., \& Widiger, T. A. (2007). Millon's dimensional model of personality disorders: a comparative study, J Personal Disord, 21, (1), 52-57.

Rossi, G., Brande, I. V., Tobac, A., Sloore, H. \& Hauben, C. (2003). Convergent validity of the MCMI-III personality disorder scales and the MMPI-2 scales. Journal of Personality Disorders, 17 (4), 330-340. 
Rossi, G., Van der Ark, L. A., \& Sloore, H. (2007). Factor analysis of the Dutch-language version of the MCMI-III. Journal of Personality Assessment, 88, 144-157.

Strack, S., \& Millon, T. (2007). Contributions to the dimensional assessment of personality disorders using Millon's model and the Millon Clinical Multiaxial Inventory (MCMI9-III). Journal of Personality Assessment, 89 (1), 5669.

Tabachnick, B., \& Fidell, L. S. (1996). Using multivariate statistics $\left(3^{\mathrm{a}}\right.$ ed.). New York: Harper Collins.

Urbina, S. (2007). Fundamentos da testagem psicológica. Porto Alegre: Artmed.
Widiger, T. A., \& Trull, T. J. (2007). Place Tectonics in the Classification of Personality Disorder: shifting to a dimensional model. American Psychologist, 62, 2, 71-83.

\section{Notas:}

1 Coordenador do Laboratório de Avaliação Psicológica e Educacional - Universidade São Francisco.

Esta pesquisa foi financiada pela FAPESP e pelo CNPq. 